\title{
Minimally invasive posterior cervical foraminotomy with tubes to prevent undesired fusion: a long-term follow-up study
}

\author{
Conor Dunn, MD, Jeffrey Moore, MD, Nikhil Sahai, MD, MPH, Kimona Issa, MD, \\ Michael Faloon, MD, Kumar Sinha, MD, Ki Soo Hwang, MD, and Arash Emami, MD \\ Seton Hall University School of Medicine, St. Joseph's Regional Medical Center, Department of Orthopaedics, Paterson, \\ New Jersey
} OBJECTIVE The objective of this study was to compare anterior cervical discectomy and fusion (ACDF) and minimally
invasive posterior cervical foraminotomy (MI-PCF) with tubes for the treatment of cervical radiculopathy in terms of the
1) overall revision proportion, 2) index and adjacent level revision rates, and 3) functional outcome scores.

METHODS The authors retrospectively reviewed the records of consecutive patients who had undergone ACDF or MI-PCF at a single institution between 2009 and 2014. Patients treated for cervical radiculopathy without myelopathy and with a minimum 2-year follow-up were compared according to the procedure performed for their pathology. Primary outcome measures included the overall rate of revision with fusion and overall revision proportion as well as the rate of index and adjacent level revisions per year. Secondarily, self-reported outcome measures-Neck Disability Index (NDI) and visual analog scale (VAS) for arm (VASa) and neck (VASn) pain-at the preoperative and postoperative evaluations were analyzed. Standard binomial and categorical comparative analyses were performed.

RESULTS Forty-nine consecutive patients were treated with MI-PCF, and 210 consecutive patients were treated with ACDF. The mean follow-up for the MI-PCF cohort was $42.9 \pm 6.6$ months (mean \pm SD) and for the ACDF cohort was $44.9 \pm 10.3$ months. There was no difference in the overall revision proportion between the two cohorts $(4[8.2 \%]$ of 49 MI-PCF vs. $12[5.7 \%]$ of 210 ACDF, $p=0.5137$ ). There was no difference in the revision rate per level per year (3.1 vs. 1.7 , respectively, $p=0.464)$. Moreover, there was no difference in the revision rate per level per year at the index level (1.8 vs. 0.7 , respectively, $p=0.4657$ ) or at an adjacent level (1.3 vs. $1.1, p=0.9056)$. Neither was there a difference between the cohorts as regards the change from preoperative to final postoperative functional outcome scores (NDI, VASa, VASn).

CONCLUSIONS Minimally invasive PCF for the treatment of cervical radiculopathy demonstrates rates of revision at the index and adjacent levels similar to those following ACDF. In order to confirm the positive efficacy and cost analysis findings in this study, future studies need to extend the follow-up and show that the rate of revision with fusion does not increase substantially over time.

https://thejns.org/doi/abs/10.3171/2018.2.SPINE171003

KEYWORDS posterior cervical foraminotomy; minimally invasive; anterior cervical discectomy and fusion; cervical radiculopathy; cervical spine; revision rate; foraminal stenosis

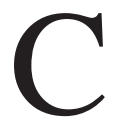

ERVICAL radiculopathy refractory to nonoperative modalities is one of the most common ailments addressed by spine surgeons today. ${ }^{18}$ Consequences of such injuries are debilitating not only to the patient but also to the healthcare system and the economy, for example, in lost productivity from employees who are on medical disability for extended periods of time. ${ }^{17}$ Since the advent of anterior discectomy and fusion (ACDF) in the 1990s and its subsequent advances, the technique has risen in popularity among spinal surgeons. ${ }^{6,20}$ Recently, however, the minimally invasive posterior approach for treating these common and debilitating defects has become a viable, if not preferred, modality. ${ }^{10,15}$

The minimally invasive posterior cervical foraminotomy (MI-PCF) was first described in cadaveric models in 2000, and several similar techniques were used before the approach with microtubes evolved., ${ }^{1,3,7,12,16}$ The open and MI-PCF have proved to be as effective as ACDF in ad-

ABBREVIATIONS ACDF = anterior cervical discectomy and fusion; MI-PCF = minimally invasive posterior cervical foraminotomy; $\mathrm{NDI}=\mathrm{Neck}$ Disability Index; RCT = randomized controlled trial; VAS = visual analog scale; VASa = VAS arm pain; VASn = VAS neck pain.

SUBMITTED September 11, 2017. ACCEPTED February 8, 2018.

INCLUDE WHEN CITING Published online June 29, 2018; DOI: 10.3171/2018.2.SPINE171003. 
dressing the symptoms of cervical radiculopathy and foraminal stenosis in several studies with short-term followups. ${ }^{8,13-15,21}$ Compared to ACDF, however, MI-PCF utilizes less hardware, shortens both the hospital length of stay and the time away from work, and reduces blood loss, medication use, and costs. , $^{2,10,11,17}$ Moreover, some investigators have hypothesized that forgoing fusion reduces a patient's risk for adjacent level disease but potentially increases their chance for subsequent revision of the index procedure. ${ }^{5,6}$ Recent studies with relatively short-term follow-ups have shown a similar rate of revision involving the index level for MI-PCF and ACDF. ${ }^{9,15}$

Specifically, MI-PCF with tubes provides a standardized approach for effectively addressing cervical radiculopathy. However, there is a natural paucity of reports in the literature confirming the long-term outcomes of the procedure. The studies that do exist have low power; thus, conclusions are guarded, warranting further investigation. In the present study, we aimed to confirm the conclusions of previous studies on MI-PCF with an emphasis on a homogeneous, minimally invasive technique with tubular dilation and a longer follow-up. Furthermore, we sought to justify the use of MI-PCF over ACDF, when appropriately indicated, by directly comparing the two approaches with regard to the overall revision proportion, revision rates at index and adjacent levels, and functional outcomes with a long-term follow-up at a single institution.

\section{Methods}

Institutional review board approval was obtained for this study. A retrospective review of our institution's database for the period from 2009 to 2014 yielded 544 consecutive patients who had undergone ACDF and 88 consecutive patients who had undergone MI-PCF. Patients were divided into two cohorts according to the procedure performed for their pathology. Criteria for study inclusion were a minimum 2-year follow-up, complete medical records, hospital stay less than 3 days, either 1 or 2 cervical operative levels, and cervical radiculopathy refractory to nonsurgical intervention. Surgical indications included a diagnosis of cervical radiculopathy without myelopathy. Myelopathy was defined by the operating surgeon, who reviewed MRI studies in conjunction with the neurological examination findings and the clinical picture. Cases with previous cervical operation were excluded.

We carefully reviewed all information from historical and physical examinations, operative notes, discharge summaries, follow-up office visits, and MRI reports. Surgical complications and failures requiring revision were noted. Patients who required revision because of persistent or new-onset radicular symptoms and with consistent radiographic imaging were stratified by level of revision including the index level or adjacent level(s). Patient demographics and other information were collected including sex, age, BMI, level(s) of operation, and smoking status.

All patients were clinically assessed postoperatively at 2 weeks, 6 weeks, 3 months, 6 months, and annually thereafter. During each clinical visit, patients were thoroughly examined, and visual analog scale (VAS) neck and arm pain and Neck Disability Index (NDI) scores were record-
TABLE 1. Summary of patient characteristics

\begin{tabular}{lccl}
\hline \multicolumn{1}{c}{ Parameter } & ACDF & Ml-PCF & p Value \\
\hline No. of patients & 210 & 49 & \\
\hline Age at surgery in yrs (SD) & $49.9(9.8)$ & $49(11.5)$ & 0.928 \\
\hline No. of F/M & $115 / 95$ & $11 / 38$ & 0.0001 \\
\hline Smoker (yes/no) & $76 / 134$ & $12 / 37$ & 0.134 \\
\hline BMl in kg/m ${ }^{2}$ & 28.4 & 28.6 & 0.821 \\
\hline Average FU in mos & 44.92 & 42.94 & 0.5276 \\
\hline Total no. of levels & 304 & 63 & \\
\hline Average no. of op levels & 1.43 & 1.29 & 0.0778 \\
\hline Location (rt/lt/bilat) & \multicolumn{3}{c}{} \\
\hline Level of operation, no. (\%) & $21 / 22 / 6$ & \\
\hline C3-4 & $6(2.0)$ & $4(6.3)$ & 0.0736 \\
\hline C4-5 & $45(14.8)$ & $3(4.8)$ & 0.038 \\
\hline C5-6 & $154(50.7)$ & $19(30.2)$ & 0.0035 \\
\hline C6-7 & $97(31.9)$ & $29(46.0)$ & 0.0408 \\
\hline C7-T1 & $2(0.7)$ & $8(12.7)$ & 0.0001 \\
\hline
\end{tabular}

$\mathrm{FU}=$ follow-up

ed. Demographic data, medical comorbidities, outcome scores, and imaging data were quantified.

\section{Operative Procedure}

Two of 4 surgeons were present to perform each MIPCF with tubes, always utilizing the same technique. Surgical side was determined by the patient's symptoms. The correct cervical level was confirmed via C-arm. A stab incision was made, followed by blunt dissection and splitting of the fascia. Sequential dilation of the soft tissue was performed with tubular dissectors. The 12-mm tube was docked at the appropriately identified level and side and its position was confirmed with fluoroscopy. Pituitary rongeurs were utilized to remove the remaining soft tissues. Next, a microscope was positioned appropriately above the tube. A high-speed bur was then utilized to thin the lateral edge of the lamina as well as the medial third of the facet joints. Once the nerve was identified, a foraminotomy was performed using 1- and 2-mm Kerrison punches until the nerve was visually free of impingement. After copious irrigation and establishment of thorough hemostasis, the tubular dissectors were removed. The fascia was closed with an absorbable Vicryl suture, and the skin was closed with a running subcuticular absorbable Monocryl suture.

\section{Statistical Analysis}

All de-identified data were inputted into an Excel spreadsheet (Microsoft Corp.). All statistical calculations were performed using GraphPad statistical software Prism version 5.01. Statistical comparisons were performed using a Z-test or Student t-test, as appropriate, to assess potential differences between proportions and means, respectively. A $p$ value less than 0.05 was considered significant.

\section{Results}

A total of 259 patients were included for the final analysis (Table 1). Two hundred ten patients, consisting 


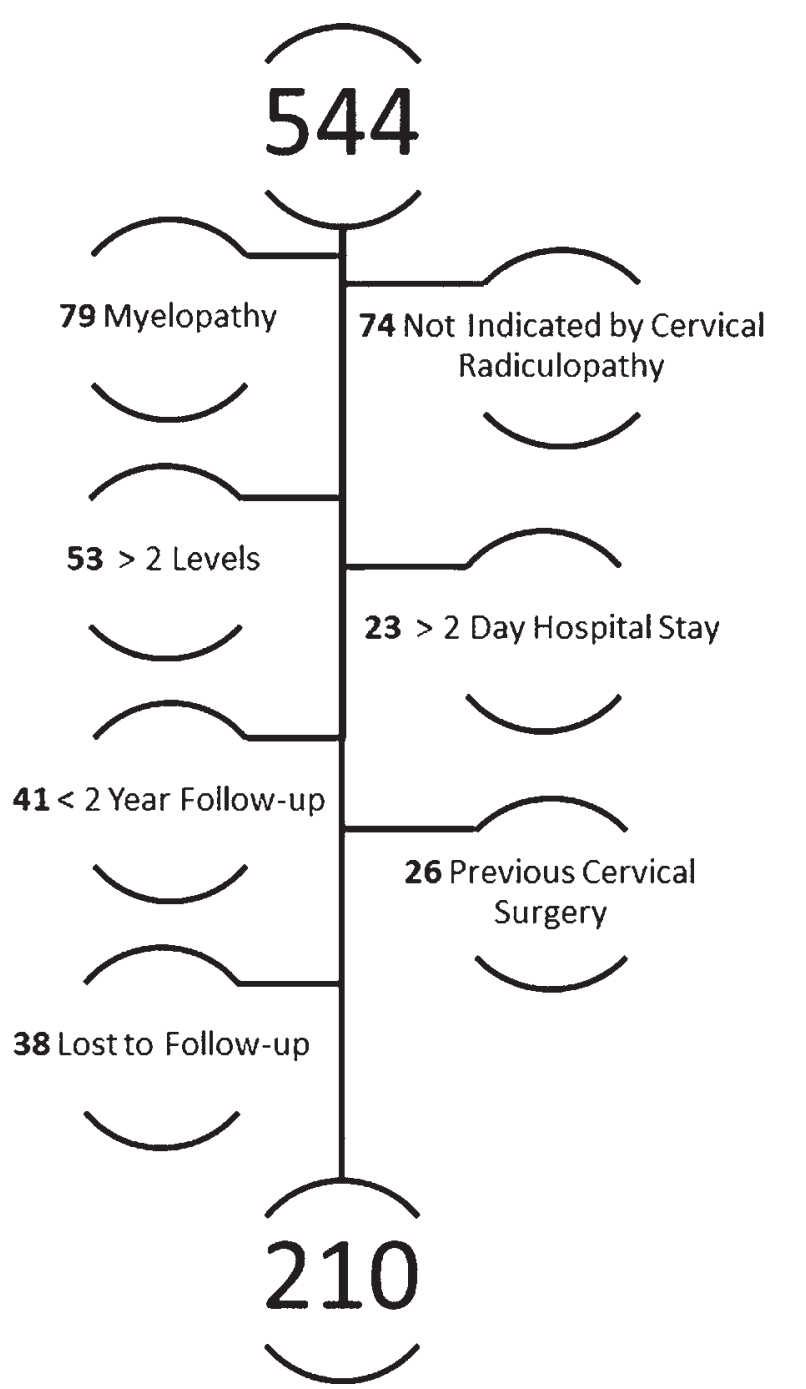

FIG. 1. Inclusion and exclusion flowchart for the ACDF cohort.

of 115 females and 95 males, with a mean age of $49.9 \pm$ 9.8 years (mean $\pm \mathrm{SD}$ ), underwent ACDF (Fig. 1). Fortynine patients, consisting of 11 females and 38 males, with a mean age of $49 \pm 11.05$ years, underwent MI-PCF (Fig. 2). Mean final follow-up for the MI-PCF cohort was 42.9 \pm 6.6 months and 44.9 \pm 10.3 months for the ACDF group. Mean age at the time of surgery, mean follow-up time, average number of operative levels, BMI, and smoking status did not differ between the cohorts. There were more men in the MI-PCF cohort than in the ACDF cohort (77.6\% vs. $45.2 \%, \mathrm{p}<0.001)$. In the ACDF cohort, there were more operations at the $\mathrm{C} 4-5(14.8 \%$ vs. $4.8 \%, \mathrm{p}=0.038)$ and C5-6 (50.7\% vs. 30.2\%, p =0.0035) levels, whereas the MI-PCF cohort had more operations at the C6-7 $(31.9 \%$ vs. $46.0 \%, \mathrm{p}=0.0408)$ and $\mathrm{C} 7-\mathrm{T} 1(0.7 \%$ vs. $12.7 \%, \mathrm{p}<$ $0.001)$ levels.

There was no difference between the cohorts in terms of age at revision surgery, revision rate at the 2-year mark, or number of complications (Table 2). At the 2-year mark postoperatively, $8(3.8 \%)$ patients in the ACDF cohort underwent revision surgery, whereas $2(4.1 \%)$ patients in the MI-PCF group underwent revision with fusion, dem-

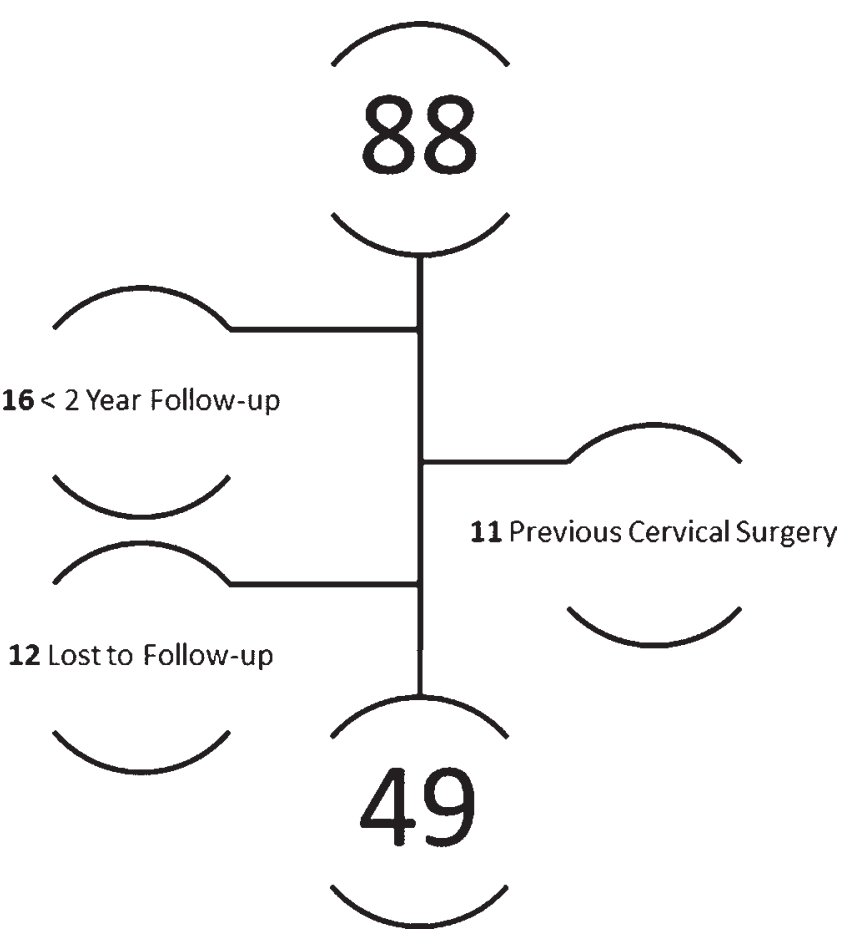

FIG. 2. Inclusion and exclusion flowchart for the MI-PCF cohort.

onstrating no statistically significant difference between the two groups $(p=1)$. Despite a larger overall proportion of revision procedures in the MI-PCF cohort than in the ACDF cohort, the difference did not reach significance ( $8.2 \%$ vs. $5.7 \%$, respectively, $\mathrm{p}=0.5137)$. The mean time to revision was 22.4 months in the ACDF cohort and 22.8 months for the MI-PCF cohort ( $\mathrm{p}=0.9767)$. Kaplan-Meier survival curves for each cohort are demonstrated in Figs. 3 and 4. There was no difference in the revision rate per level per year between the MI-PCF and ACDF cohorts (3.1 vs 1.7, respectively, $p=0.464$; Table 2 ). Likewise, there was no difference in the revision rate per level per year at the index level (1.8 vs 0.7 , respectively, $\mathrm{p}=0.4657$ ) or at an adjacent level (1.3 vs 1.1, respectively, $\mathrm{p}=0.9056)$.

All 4 revisions in the MI-PCF cohort were treated with an ACDF. Fusion procedures were indicated by recurrence and progression of symptoms along with further degenerative changes of the index level, including foraminal and central stenosis. Three of the revision fusions included adjacent levels due to progression of degeneration at these levels. Five of the ACDF revisions (41.7\% of revisions, $2.4 \%$ of ACDF cases) were indicated by pseudarthrosis of the initial fusion, 5 were indicated by adjacent segment disease, and 2 (16.7\% of revisions, $0.95 \%$ of ACDF cases) were due to traumatic injuries causing recurrent herniation of an adjacent level. There was 1 case of superficial wound infection in the ACDF cohort for which the patient was brought back to the operating room for a washout and was successfully treated with antibiotics. There were 2 cases of instrumentation failure -1 in which a screw backed out of position and required reoperation and repositioning of the screw, and 1 in which the plate loosened and required reoperation and repositioning of the plate-both of which achieved successful outcomes. 
TABLE 2. Operative results

\begin{tabular}{lccl}
\hline \multicolumn{1}{c}{ Parameter } & ACDF & Ml-PCF & p Value \\
\hline Age at reoperation in yrs (SD) & $46.6(6.6)$ & $46.9(10.3)$ & 0.9518 \\
\hline Complications, no. (\%) & $7(3.3)$ & 0 & 1 \\
\hline Revision 2 yrs, no. (\%) & $8(3.8)$ & $2(4.1)$ & 1 \\
\hline Total no. revised & 12 & 4 & \\
\hline Revision proportion, \% & 5.7 & 8.2 & 0.5137 \\
\hline No. levels revised & 19 & 7 & \\
\hline Revision rate per level per yr & 1.7 & 3.1 & 0.464 \\
\hline Covering index level, no. & 7 & 4 & \\
\hline Revision rate per index level per yr & 0.7 & 1.8 & 0.4657 \\
\hline $\begin{array}{l}\text { Covering adjacent level, no. } \\
\text { Revision rate per adjacent level } \\
\text { per yr }\end{array}$ & 12 & 3 & \\
\hline TTR in mos & 1.1 & 1.3 & 0.9056 \\
\hline
\end{tabular}

TTR = time to revision

Functional outcome scores are shown in Table 3. Baseline NDI, VASn, and VASa scores for the MI-PCF cohort were $34.2 \pm 13.3,6.3 \pm 3.4$, and $5.8 \pm 3.6$, respectively. Similarly, the ACDF group mean scores were $35.6 \pm 17.6$, $6.8 \pm 3.9$, and $5.9 \pm 3.8$, respectively. There was no difference in NDI, VASn, or VASa scores between the cohorts at baseline ( $\mathrm{p}=0.6016,0.4091,0.8671$, respectively) or the final follow-up $(\mathrm{p}=0.8922,0.1013,0.200)$.

Table 4 allows comparison of the characteristics of patients requiring revision in each cohort with those of the patients who did not undergo revision. In the ACDF cohort, sex, age, smoking status, BMI, and operative level did not show statistical significance in a comparison between revision and nonrevision patients. In the MI-PCF group, patients who smoked underwent significantly more revisions than those who did not $(3[25 \%]$ vs. 0 [0\%], p < $0.001)$. There were $13(26.5 \%)$ MI-PCF procedures that were 2 levels, and $1(7.7 \%)$ was revised at 26 months.

\section{Discussion}

The success of ACDF since its advent in the 1990s has caused it to become the gold-standard treatment for most cases of cervical radiculopathy. Despite its major advantages, ACDF comes with now well-described complica-

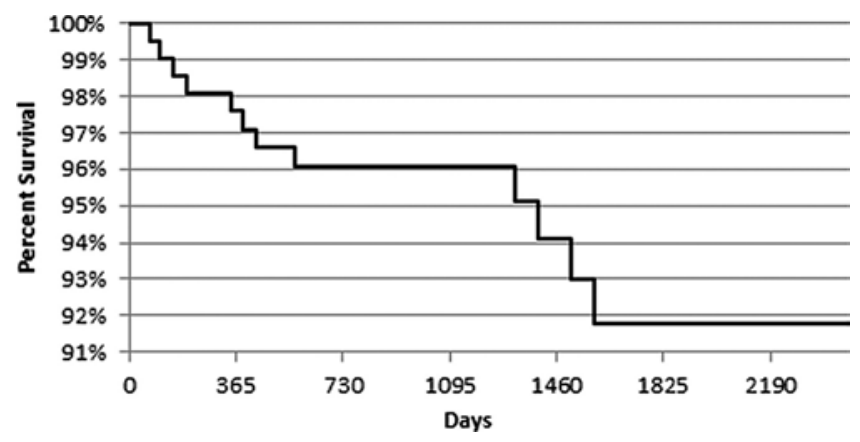

FIG. 3. Kaplan-Meier survival curve for the ACDF cohort with a mean follow-up of 44.9 months.

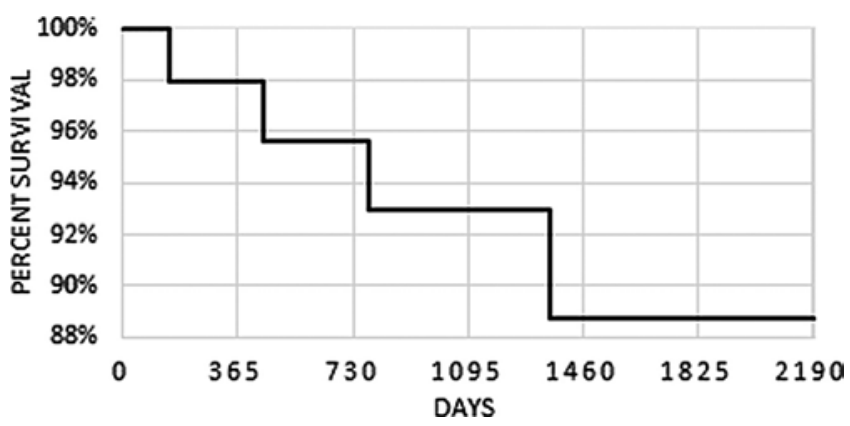

FIG. 4. Kaplan-Meier survival curve for the MI-PCF cohort with a mean follow-up of 42.9 months.

tions including graft site problems, pseudarthrosis, access complications, and adjacent segment degeneration. Minimally invasive PCF, while unable to address central pathology and other indications for ACDF, has proved as effective as ACDF in select patients, with several distinct advantages. Moreover, MI-PCF has grown in scope to address not only unilateral single-level foraminal stenosis with cervical radiculopathy but also two-level symptoms with or without lateral discectomy.

Naturally, the study of a PCF-treated cohort with the longest follow-up has involved the open technique. In 2009, Jagannathan et al. described successful results in 162 patients treated by a sole surgeon, with a minimum follow-up of 5 years. ${ }^{8}$ In a study spanning 24 years at a single institution with many participating surgeons, Bydon et al. treated patients with open PCF and an average final follow-up of 4.15 years. ${ }^{4}$ Long-term resolution of symptoms was achieved in $85 \%$ of the patients in that series. Fifteen of the 151 patients underwent revision, for a revision proportion similar to ours at 3.6 years $(9.9 \%$ vs $8.2 \%)$. The difference in the proportions may be attributable to the 6-month longer follow-up in our series or to our use of minimally invasive tube dilation in our surgical technique. Interestingly, Bydon et al. noted that those least likely to undergo revision were those with no preoperative neck pain. In our series, surgeons may have preferentially offered ACDF to patients with increased neck pain, potentially decreasing our revisions with fusion.

In Adamson's landmark description of microendoscopic posterior cervical laminoforaminotomy in 2001, 97 of 100 patients obtained excellent or good results and were able to return to their preoperative employment and baseline level of physical activity with a minimum of 6 months' follow-up. ${ }^{1}$ The mean return to work was 1.9 weeks, with 60 patients able to return to work in a week or less and all going home the same or next day after surgery. Additionally, 84 patients did not require any additional prescriptions for pain medication following the initial 7-day supply received postoperatively.

One of the greatest advantages proposed for PCF is its ability to retain motion at the operated segment, theoretically reducing the rate of adjacent segment degeneration and revision procedures that are a common and known complication of ACDF. ${ }^{5,6}$ The impact of retaining motion at the operated segment is to prevent increased motion at the adjacent levels, which is seen in ACDF, leading to an increased iatrogenic adjacent segment degeneration rate. 
TABLE 3. Functional outcome scores

\begin{tabular}{|c|c|c|c|c|c|c|c|c|}
\hline \multirow[b]{2}{*}{ Scale } & \multicolumn{3}{|c|}{ Preop Scores } & \multicolumn{3}{|c|}{ Final Scores } & \multicolumn{2}{|c|}{ Score Change } \\
\hline & ACDF & MI-PCF & $p$ Value & ACDF & MI-PCF & $p$ Value & ACDF & MI-PCF \\
\hline NDI & $35.6(17.6)$ & $34.2(13.3)$ & 0.6016 & $9.7(4.8)$ & $9.6(3.9)$ & 0.8922 & 25.9 & 24.6 \\
\hline VASn & $6.8(3.9)$ & $6.3(3.4)$ & 0.4091 & $1.4(0.8)$ & $1.2(0.6)$ & 0.1013 & 5.4 & 5.1 \\
\hline VASa & $5.9(3.8)$ & $5.8(3.6)$ & 0.8671 & $0.6(0.3)$ & $0.4(0.3)$ & 0.200 & 5.4 & 5.4 \\
\hline
\end{tabular}

Values expressed as the mean (standard deviation), unless indicated otherwise.

As hypothesized, MI-PCF has shown a low fusion rate of the adjacent level, at $0.9 \%$ per adjacent level per year in the literature. ${ }^{15}$ Similarly, our rate of fusion per adjacent level per year was just $1.1 \%$.

In a series of 70 patients undergoing MI-PCF, Skovrlj et al. demonstrated a $7.1 \%$ reoperation rate over a 32-month follow-up..$^{15}$ Additionally, these authors included multilevel and bilateral procedures and had a distribution of operated levels similar to ours with C6-7 being the most frequent level of operation $(48.4 \%$ vs. $46.0 \%$, respectively). Our study had a rate of revision per index level per year of $1.8 \%$, whereas Skovrlj et al.'s was $1.1 \%$. The difference between the two rates may be explained by our longer follow-up as one of our patients revised to ACDF at the index level was more than 4.5 years removed from the initial MI-PCF. Additionally, our proportion of revisions at 43 months of follow-up at $8.2 \%$ is consistent with their findings.

Despite extensive research and publications on both ACDF and MI-PCF, only two studies have directly compared clinical results between the approaches, and neither has described utilizing a homogeneous MI-PCF technique with tubes for decompression. In 2008, Ruetten et al. published a randomized controlled trial (RCT) that included 89 minimally invasive endoscopic PCFs with a 2-year follow-up in patients with lateral cervical disc herniations and radicular arm pain. ${ }^{13}$ The authors found no difference between the patients who underwent $\operatorname{ACDF}(n=86)$ and those who underwent PCF in terms of revision $(4.7 \%$ vs. $6.7 \%$, respectively), clinical outcome scores, resolution of neurological or radicular symptoms, and complications. These 2-year revision rates were similar to ours for ACDF and MI-PCF (3.8\% and 4.1\%, respectively). A propensitymatched analysis of ACDF and MI-PCF by Lubelski et al. in 2015 revealed no difference in reoperation rates at the index level between the two cohorts at the 2-year postoperative visit (9 [4.8\%] of 188 ACDFs vs 9 [6.4\%] of 140 MI-PCFs, $p=0.7) .{ }^{9}$ In a separate study, Wang et al. found a similar 5\% conversion to ACDF from MI-PCF among a cohort of 178 patients and a follow-up averaging 31.7 months. $^{19}$

Cost analysis studies in three studies have confirmed the short-term cost savings of MI-PCF over ACDF. In 2014, after retrospectively analyzing 101 patients, Mansfield et al. demonstrated that ACDF costs $89 \%$ more than MI-PCF in patients with cervical radiculopathy. ${ }^{11}$ This study did not include follow-up, however, and the authors concluded that the initial substantial difference in cost was related to the cost of the implant for ACDF. The results did not appear to be affected by patient sex or smoking status. In a retrospective 1-year cost-utility analysis in patients with single-level cervical radiculopathy, Alvin et al. revealed that, although both cohorts had achieved good clinical improvements, only MI-PCF was cost-effective at the $\$ 100,000$ per quality-adjusted life year (QALY) at 1-year postoperatively ( $\$ 131,951 / \mathrm{QALY}$ gained vs $\$ 79,856$ / QALY gained). ${ }^{2}$ The authors stressed the need for future long-term cost-utility analysis studies. Moreover, in 2010, Tumialán et al. reviewed the cost-effectiveness of both procedures in active-duty military personnel. ${ }^{17}$ Return to work with successful treatment had a total cost ranging from $\$ 20,094$ to $\$ 30,553$ more in the ACDF group than in the PCF group. The majority of savings was found in the disparity in the return to active duty as the ACDF cohort

TABLE 4. Revision patients

\begin{tabular}{|c|c|c|c|c|c|c|}
\hline \multirow[b]{2}{*}{ Parameter } & \multicolumn{3}{|c|}{ ACDF } & \multicolumn{3}{|c|}{ MI-PCF } \\
\hline & Revision & No Revision & $\mathrm{p}$ Value & Revision & No Revision & $p$ Value \\
\hline Male & 5 & 90 & 1 & 3 & 35 & 1 \\
\hline Smoker & 3 & 73 & 0.5427 & 3 & 9 & 0.0009 \\
\hline Age in yrs (SD) & $46.6(6.6)$ & $50.2(9.9)$ & 0.2196 & $46.9(10.3)$ & $49.2(11.7)$ & 0.6988 \\
\hline $\mathrm{BMI}$ in $\mathrm{kg} / \mathrm{m}^{2}(\mathrm{SD})$ & $27.3(5.1)$ & $28.5(5.1)$ & 0.4676 & $29.2(6.5)$ & $28.6(4.8)$ & 0.7962 \\
\hline \multicolumn{7}{|c|}{ Level of operation, no. (\%) } \\
\hline C3-4 & $1(16.7)$ & $5(83.3)$ & 0.3198 & 0 & 4 & 1 \\
\hline C4-5 & $2(4.4)$ & $43(95.6)$ & 1 & 0 & 3 & 1 \\
\hline C5-6 & $9(5.8)$ & $145(94.2)$ & 0.6518 & $1(5.3)$ & $18(94.7)$ & 1 \\
\hline C6-7 & $8(8.2)$ & $89(91.8)$ & 0.466 & $2(6.9)$ & 27 (93.1) & 0.6441 \\
\hline $\mathrm{C} 7-\mathrm{T} 1$ & 0 & $2(100)$ & 1 & $1(12.5)$ & $7(87.5)$ & 0.4274 \\
\hline
\end{tabular}


took a mean of 14.8 weeks longer to resume work than the MI-PCF cohort. Therefore, the literature strongly suggests that MI-PCF reduces overall costs in short-term studies and the return to work time.

In our study, to the best of our knowledge, the follow-up was the longest to date for any MI-PCF technique directly compared to ACDF. This study also adds value to our understanding of the outcomes of MI-PCF by evaluating long-term functional outcome scores in addition to revision rates. While the literature has demonstrated superior short-term functional outcome scores with MI-PCF, it has been unclear how these scores change with a longer follow-up. We found no difference in the functional outcome scores or revision proportions with nearly 43 months' average follow-up in the MI-PCF cohort. However, the rising revision rate in proportion to $\mathrm{ACDF}$ is concerning and leaves open the possibility of an unacceptably high revision rate and cost analysis with poorer functional outcome scores at the 10-year mark.

There were several limitations to this study. Being a retrospective study rather than a RCT, patients with central disc herniations and other pathologies such as degenerative listhesis were likely to have undergone an ACDF without indication for MI-PCF but were included for the final analysis. While this retrospective limitation affects the comparability of the cohorts, other available studies in the literature are also retrospective, and our revision rate in the ACDF cohort was consistent with those in the literature as well. We attempted to better match the cohorts by removing many patients from the $\mathrm{ACDF}$ cohort who likely would not have been offered MI-PCF, that is, patients with myelopathy, with fusions spanning more than 2 levels, with hospital stays longer than 2 days, and without cervical radiculopathy refractory to conservative treatment. While our cohorts were similar in age, BMI, proportion of smokers, and follow-up length, there were a greater proportion of men in the MI-PCF group and a preference of surgical level between the cohorts. Analysis of revision by cervical level did not demonstrate a risk in either cohort, however, thus validating the comparability of the cohorts. Patients who underwent reoperation at an outside institution would have been missed. There is no reason to expect a difference in missed reoperations at outside institutions between the ACDF and MI-PCF cohort, however.

\section{Conclusions}

With a long-term follow-up averaging nearly 43 months, this study demonstrated that MI-PCF with tubes for the treatment of cervical radiculopathy is a safe and effective minimally invasive technique with rare complications and a revision rate and functional outcome scores similar to those for ACDF. Further studies need to extend the longterm follow-up and the cost analysis to confirm that the revision rate does not increase to an unacceptable level at 10 years, affecting the cost-effectiveness of the procedure.

\section{References}

1. Adamson TE: Microendoscopic posterior cervical laminoforaminotomy for unilateral radiculopathy: results of a new technique in 100 cases. J Neurosurg 95 (1 Suppl):51-57, 2001
2. Alvin MD, Lubelski D, Abdullah KG, Whitmore RG, Benzel EC, Mroz TE: Cost-utility analysis of anterior cervical discectomy and fusion with plating (ACDFP) versus posterior cervical foraminotomy (PCF) for patients with single-level cervical radiculopathy at 1-year follow-up. Clin Spine Surg 29:E67-E72, 2016

3. Burke TG, Caputy A: Microendoscopic posterior cervical foraminotomy: a cadaveric model and clinical application for cervical radiculopathy. J Neurosurg 93 (1 Suppl):126-129, 2000

4. Bydon M, Mathios D, Macki M, de la Garza-Ramos R, Sciubba DM, Witham TF, et al: Long-term patient outcomes after posterior cervical foraminotomy: an analysis of 151 cases. J Neurosurg Spine 21:727-731, 2014

5. Cho TG, Kim YB, Park SW: Long term effect on adjacent segment motion after posterior cervical foraminotomy. Korean J Spine 11:1-6, 2014

6. DePalma AF, Rothman RH, Lewinnek GE, Canale ST: Anterior interbody fusion for severe cervical disc degeneration. Surg Gynecol Obstet 134:755-758, 1972

7. Fessler RG, Khoo LT: Minimally invasive cervical microendoscopic foraminotomy: an initial clinical experience. Neurosurgery 51 (5 Suppl):S37-S45, 2002

8. Jagannathan J, Sherman JH, Szabo T, Shaffrey CI, Jane JA Sr: The posterior cervical foraminotomy in the treatment of cervical disc/osteophyte disease: a single-surgeon experience with a minimum of 5 years' clinical and radiographic followup. J Neurosurg Spine 10:347-356, 2009

9. Lubelski D, Healy AT, Silverstein MP, Abdullah KG, Thompson NR, Riew KD, et al: Reoperation rates after anterior cervical discectomy and fusion versus posterior cervical foraminotomy: a propensity-matched analysis. Spine J 15:1277-1283, 2015

10. Lubelski D, Mihalovich KE, Skelly AC, Fehlings MG, Harrop JS, Mummaneni PV, et al: Is minimal access spine surgery more cost-effective than conventional spine surgery? Spine (Phila Pa 1976) 39 (22 Suppl 1):S65-S74, 2014

11. Mansfield HE, Canar WJ, Gerard CS, O'Toole JE: Single-level anterior cervical discectomy and fusion versus minimally invasive posterior cervical foraminotomy for patients with cervical radiculopathy: a cost analysis. Neurosurg Focus 37(5):E9, 2014

12. Roh SW, Kim DH, Cardoso AC, Fessler RG: Endoscopic foraminotomy using MED system in cadaveric specimens. Spine (Phila Pa 1976) 25:260-264, 2000

13. Ruetten S, Komp M, Merk H, Godolias G: Full-endoscopic cervical posterior foraminotomy for the operation of lateral disc herniations using 5.9-mm endoscopes: a prospective, randomized, controlled study. Spine (Phila Pa 1976) 33:940-948, 2008

14. Ruetten S, Komp M, Merk H, Godolias G: A new fullendoscopic technique for cervical posterior foraminotomy in the treatment of lateral disc herniations using 6.9-mm endoscopes: prospective 2-year results of 87 patients. Minim Invasive Neurosurg 50:219-226, 2007

15. Skovrlj B, Gologorsky Y, Haque R, Fessler RG, Qureshi SA: Complications, outcomes, and need for fusion after minimally invasive posterior cervical foraminotomy and microdiscectomy. Spine J 14:2405-2411, 2014

16. Terai H, Suzuki A, Toyoda H, Yasuda H, Kaneda K, Katsutani $\mathrm{H}$, et al: Tandem keyhole foraminotomy in the treatment of cervical radiculopathy: retrospective review of 35 cases. $\mathbf{J}$ Orthop Surg Res 9:38, 2014

17. Tumialán LM, Ponton RP, Gluf WM: Management of unilateral cervical radiculopathy in the military: the cost effectiveness of posterior cervical foraminotomy compared with anterior cervical discectomy and fusion. Neurosurg Focus 28(5):E17, 2010

18. van Geest S, Kuijper B, Oterdoom M, van den Hout W, Brand 
R, Stijnen T, et al: CASINO: surgical or nonsurgical treatment for cervical radiculopathy, a randomised controlled trial. BMC Musculoskelet Disord 15:129, 2014

19. Wang TY, Lubelski D, Abdullah KG, Steinmetz MP, Benzel EC, Mroz TE: Rates of anterior cervical discectomy and fusion after initial posterior cervical foraminotomy. Spine J 15:971-976, 2015

20. Wirth FP, Dowd GC, Sanders HF, Wirth C: Cervical discectomy. A prospective analysis of three operative techniques. Surg Neurol 53:340-348, 2000

21. Woertgen C, Rothoerl RD, Henkel J, Brawanski A: Long term outcome after cervical foraminotomy. J Clin Neurosci 7:312-315, 2000

\section{Disclosures}

Dr. Faloon is a consultant for and member of the speaker's bureau of Depuy and K2M.

\section{Author Contributions}

Conception and design: Emami, Hwang. Acquisition of data: Dunn, Moore, Sahai. Analysis and interpretation of data: Moore, Sahai, Issa. Drafting the article: Dunn, Moore, Sahai. Critically revising the article: Issa, Faloon. Reviewed submitted version of manuscript: Sinha. Statistical analysis: Hwang. Study supervision: Emami, Faloon, Sinha, Hwang.

\section{Supplemental Information}

Previous Presentations

Portions of this work have been presented as a podium presentation at the Eastern Orthopaedic Association Annual Meeting held in Miami Beach, FL, on October 18-21, 2017.

\section{Correspondence}

Arash Emami: University Spine Center, Wayne, NJ. emamiresearch@gmail.com. 